\title{
Clinical significance versus statistical significance
}

Reference 4 in this Endgames article (BMJ 2014;348:g2130, doi:10.1136/bmj.g2130) should have had the citation $B M J$ 2013;347:f6282 and link to http://www.bmj.com/content/347/ bmj.f6282. Instead, this reference mistakenly listed the citation for a correction to that article (BMJ 2013;347:f6441) and linked to the correction as well.

Cite this as: BMJ 2014;348:94552

๑ BMJ Publishing Group Ltd 2014 